\title{
WEAKLY COMPACT OPERATORS ON KÖTHE-BOCHNER SPACES WITH THE MIXED TOPOLOGY
}

\author{
KRZYSZTOF FELEDZIAK \\ Faculty of Mathematics, Computer Science and Econometrics, University of Zielona Góra \\ Szafrana 4a, 65-516 Zielona Góra, Poland \\ E-mail: K.Feledziak@wmie.uz.zgora.pl
}

\begin{abstract}
Let $E$ be a Banach function space and let $X$ be a real Banach space. We examine weakly compact linear operators from a Köthe-Bochner space $E(X)$ endowed with some natural mixed topology $\gamma_{E(X)}$ (in the sense of Wiweger) to a Banach space $Y$.
\end{abstract}

1. Introduction and preliminaries. The paper is devoted to the study of weakly compact operators acting from a Köthe-Bochner space $E(X)$ endowed with the mixed topology $\gamma_{E(X)}$ to a Banach space $Y$.

The problem of characterizing weakly compact operators has been considered by many authors (see [Ga], [G], [D], [Ru]). In [G, III. 3, Corollary 1 of Theorem 11] A. Grothendieck has proved that every linear continuous operator from a quasinormable Hausdorff locally convex space $X$ into a Banach space $Y$, which transforms bounded sets into relatively weakly compact sets is weakly compact. Grothendieck's result was partially extended by D. van Dulst to the case when $Y$ is Fréchet space, but under the additional assumption about the space $X$ (see [D] for more details). Both those results were later extended by W. Ruess in $[\mathrm{Ru}]$ for operators acting between gDF-spaces and Fréchet spaces. gDF spaces have been introduced and studied by K. Noureddine in $\left[\mathrm{No}_{1}\right],\left[\mathrm{No}_{2}\right]$ under the name of $D_{b}$-spaces ("espaces $D_{b}$ " in French). Since not only many properties of classical DF-spaces of Grothendieck carry over to $D_{b}$-spaces, but also some fruitful DF-techniques can be applied to them, W. Ruess decided in [Ru] to change their original name of Noureddine to the gDF-spaces (generalized DF-spaces), in order to stress their close relationship with DF-spaces.

2000 Mathematics Subject Classification: 47B38, 46E40, 46E30.

Key words and phrases: Köthe-Bochner spaces, mixed topologies, weakly compact operators, DF-spaces, gDF-spaces, conditional weak compactness, weak compactness, almost reflexivity.

The paper is in final form and no version of it will be published elsewhere. 
Given a topological vector space $(L, \xi)$, by $(L, \xi)^{*}$ or $L_{\xi}^{*}$ we will denote its topological dual and by $B d(L, \xi)$ we will denote the family of all $\xi$-bounded subsets of $L$. We denote by $\sigma(L, K)$ and $\tau(L, K)$ the weak topology and the Mackey topology on $L$ with respect to a dual system $\langle L, K\rangle$ respectively. Recall that a subset $Z$ of $L$ is said to be conditionally $\sigma(L, K)$-compact (resp. relatively $\sigma(L, K)$-sequentially compact) whenever each sequence in $Z$ contains a $\sigma(L, K)$-Cauchy subsequence (resp. each sequence in $Z$ contains a subsequence which is $\sigma(L, K)$-convergent to some element of $L)$.

For terminology concerning Riesz spaces and function spaces we refer to [AB], [KA]. Throughout the paper let $(\Omega, \Sigma, \mu)$ be an atomless, complete and $\sigma$-finite measure space and let $L^{0}$ denote the corresponding space of $\mu$-equivalence classes of $\Sigma$-measurable real valued functions. Then $L^{0}$ is super Dedekind complete Riesz space under the ordering $u_{1} \leq u_{2}$ whenever $u_{1}(\omega) \leq u_{2}(\omega)$ a.e. on $\Omega$. A Banach space $\left(E,\|\cdot\|_{E}\right)$ is assumed to be a Banach function space, that is, $E$ is an ideal of $L^{0}$ with $\operatorname{supp} E=\Omega$ and $\|\cdot\|_{E}$ is a Riesz norm. The Köthe dual of $E$ is defined by

$$
E^{\prime}=\left\{v \in L^{0}: \int_{\Omega}|u(\omega) v(\omega)| d \mu<\infty \text { for all } u \in E\right\} .
$$

The associated norm $\|\cdot\|_{E^{\prime}}$ on $E^{\prime}$ is defined for $v \in E^{\prime}$ by

$$
\|v\|_{E^{\prime}}=\sup \left\{\left|\int_{\Omega} u(\omega) v(\omega) d \mu\right|: u \in E,\|u\|_{E} \leq 1\right\} .
$$

It is known that supp $E^{\prime}=\Omega$ (see [KA, Theorem 6.1.3]). Recall that a Banach function space $\left(E,\|\cdot\|_{E}\right)$ is said to be perfect if $E^{\prime \prime}=E$ and $\|u\|_{E^{\prime \prime}}=\|u\|_{E}$ for $u \in E$. It is well known that $E$ is perfect if and only if the norm $\|\cdot\|_{E}$ satisfies both the $\sigma$-Fatou property (i.e., $0 \leq u_{n} \uparrow u$ in $E$ implies $\left\|u_{n}\right\|_{E} \uparrow\|u\|_{E}$ ) and the $\sigma$-Levy property (i.e., if $0 \leq u_{n} \uparrow$ in $E$ and $\sup \left\|u_{n}\right\|_{E}<\infty$, then there exists $u$ in $E$ such that $u_{n} \uparrow u$ ) (see [KA, Theorem 6.1.7]). We denote by $E_{a}$ the ideal of elements of order continuous norm in $E$, i.e.,

$$
E_{a}=\left\{u \in E:|u| \geq u_{n} \downarrow 0 \text { in } E \text { imply }\left\|u_{n}\right\| \rightarrow 0\right\} .
$$

From now on in this paper we assume that $\left(E,\|\cdot\|_{E}\right)$ is a perfect Banach function space and $\operatorname{supp}\left(E^{\prime}\right)_{a}=\Omega$. Note that $\left(L^{1}\right)^{\prime}=L^{\infty}$ and $\left(L^{\infty}\right)_{a}=\{0\}$. Hence the space $L^{1}$ is excluded.

Let $Z$ be a $\sigma\left(E,\left(E^{\prime}\right)_{a}\right)$-bounded subset of $E$. Then $Z$ is also $|\sigma|\left(E,\left(E^{\prime}\right)_{a}\right)$-bounded (see $\left[\mathrm{AB}\right.$, Theorem 2.33]), so one can define a Riesz seminorm $p_{Z}$ on $\left(E^{\prime}\right)_{a}$ by

$$
p_{Z}(v)=\sup \left\{\int_{\Omega}|u(\omega) v(\omega)| d \mu: u \in Z\right\} .
$$

Then by $\left[\mathrm{N}_{2}\right.$, Theorem 1.1] we have

$$
B d\left(E, \sigma\left(E,\left(E^{\prime}\right)_{a}\right)\right)=B d\left(E,\|\cdot\|_{E}\right) .
$$

Hence for every $\sigma\left(E,\left(E^{\prime}\right)_{a}\right)$-bounded subset $Z$ of $E$ the seminorm $p_{Z}$ on $\left(E^{\prime}\right)_{a}$ is order continuous. Making use of $\left[\mathrm{N}_{4}\right.$, Proposition 1.1] and [BD, Corollary 5.2] we get

Proposition 1.1. For a subset $Z$ of $E$ the following statements are equivalent:

(i) $Z$ is conditionally $\sigma\left(E,\left(E^{\prime}\right)_{a}\right)$-compact.

(ii) $Z$ is relatively $\sigma\left(E,\left(E^{\prime}\right)_{a}\right)$-sequentially compact. 
(iii) $Z$ is $\sigma\left(E,\left(E^{\prime}\right)_{a}\right)$-bounded.

(iv) $Z$ is $\|\cdot\|_{E}$-bounded.

Now we establish terminology and some basic results concerning vector-valued function spaces (see $\left[\mathrm{Bu}_{1}\right],\left[\mathrm{Bu}_{2}\right],[\mathrm{L}$, Chap. 3$\left.]\right)$. Let $\left(X,\|\cdot\|_{X}\right)$ be a real Banach space and let $X^{*}$ stand for the Banach dual of $X$. Let $B_{X}$ denote the closed unit ball in $X$. By $L^{0}(X)$ we denote the set of $\mu$-equivalence classes of all strongly $\Sigma$-measurable functions $f: \Omega \rightarrow X$. The $F$-norm

$$
\|f\|_{L^{0}(X)}=\int_{\Omega} \frac{\|f(\omega)\|_{X}}{1+\|f(\omega)\|_{X}} w(\omega) d \mu \quad \text { for } \quad f \in L^{0}(X),
$$

where $w: \Omega \rightarrow(0, \infty)$ is a $\Sigma$-measurable function with $\int_{\Omega} w(\omega) d \mu=1$, determines the topology $\mathcal{T}_{0}(X)$ on $L^{0}(X)$ of convergence in measure on sets of finite measure.

For $f \in L^{0}(X)$ let us set $\tilde{f}(\omega)=\|f(\omega)\|_{X}$ for $\omega \in \Omega$. The linear space

$$
E(X)=\left\{f \in L^{0}(X): \tilde{f} \in E\right\}
$$

provided with the norm $\|f\|_{E(X)}:=\|\widetilde{f}\|_{E}$ is a Banach space and is called a Köthe-Bochner space (see $[\mathrm{L}])$. For $r>0$ we will write

$$
B_{E(X)}(r)=\left\{f \in E(X):\|f\|_{E(X)} \leq r\right\} .
$$

Let $L^{0}\left(X^{*}, X\right)$ be the set of weak*-equivalence classes of all weak ${ }^{*}$-measurable functions $g: \Omega \rightarrow X^{*}$. One can define the so-called abstract norm $\vartheta: L^{0}\left(X^{*}, X\right) \rightarrow L^{0}$ by $\vartheta(g)=\sup \left\{\left|g_{x}\right|: x \in B_{X}\right\}$, where $g_{x}(\omega)=g(\omega)(x)$ for $\omega \in \Omega$ and $x \in X$. Then for $f \in L^{0}(X)$ and $g \in L^{0}\left(X^{*}, X\right)$ the function $\langle f, g\rangle: \Omega \rightarrow \mathbb{R}$ defined by $\langle f, g\rangle(\omega)=\langle f(\omega), g(\omega)\rangle$ is measurable and $|\langle f, g\rangle| \leq \widetilde{f} \vartheta(g)$. Moreover, $\vartheta(g)=\widetilde{g}$ for $g \in L^{0}\left(X^{*}\right)$. For an ideal $M$ of $E^{\prime}$ let

$$
M\left(X^{*}, X\right)=\left\{g \in L^{0}\left(X^{*}, X\right): \vartheta(g) \in M\right\} .
$$

Then $M\left(X^{*}, X\right)$ is an ideal of $E^{\prime}\left(X^{*}, X\right)$, i.e., if $\vartheta\left(g_{1}\right) \leq \vartheta\left(g_{2}\right)$ with $g_{1} \in E^{\prime}\left(X^{*}, X\right)$ and $g_{2} \in M\left(X^{*}, X\right)$, then $g_{1} \in M\left(X^{*}, X\right) . M\left(X^{*}, X\right)$ can be provided with the norm

$$
\|g\|_{M\left(X^{*}, X\right)}:=\|\vartheta(g)\|_{E^{\prime}} \quad \text { for } g \in M\left(X^{*}, X\right) .
$$

In particular, we will consider the dual pair $\left\langle E(X),\left(E^{\prime}\right)_{a}\left(X^{*}, X\right)\right\rangle$ with the duality:

$$
\langle f, g\rangle=\int_{\Omega}\langle f(\omega), g(\omega)\rangle d \mu \quad \text { for } f \in E(X), g \in\left(E^{\prime}\right)_{a}\left(X^{*}, X\right) .
$$

2. Mixed topologies on Köthe-Bochner spaces. In this section we consider the mixed topology $\gamma\left[\mathcal{T}_{E}(X),\left.\mathcal{T}_{0}(X)\right|_{E(X)}\right]$ on $E(X)$ (briefly $\left.\gamma_{E(X)}\right)$, where $\mathcal{T}_{E}(X)$ stands for the topology on $E(X)$ of the norm $\|\cdot\|_{E(X)}$. For the definition and basic properties of $\gamma_{E(X)}$ see $[\mathrm{F}],[\mathrm{W}]$. In case when $X=\mathbb{R}$ the mixed topology $\gamma_{E}\left(=\gamma_{E(\mathbb{R})}\right)$ on $E$ has been studied in $\left[\mathrm{N}_{1}\right]$ and $\left[\mathrm{N}_{2}\right]$. It is known that $\left.\mathcal{T}_{0}(X)\right|_{E(X)} \subset \gamma_{E(X)} \subset \mathcal{T}_{E}(X)$. We will need the following:

Proposition 2.1. We have $B d\left(E(X), \gamma_{E(X)}\right)=B d\left(E(X),\|\cdot\|_{E(X)}\right)$.

Proof. Using [KA, Lemma 4.3.4] we see that $B_{E(X)}(1)$ is closed in $\left(E(X),\left.\mathcal{T}_{0}(X)\right|_{E(X)}\right)$. Hence by [W, Theorem 2.4.1] we get $B d\left(E(X), \gamma_{E(X)}\right)=B d\left(E(X),\|\cdot\|_{E(X)}\right)$. 
The mixed topology $\gamma_{E(X)}$ is a Hausdorff locally convex-solid topology on $E(X)$ (see $[\mathrm{F}, \S 3])$ and it is the finest locally convex topology on $E(X)$ which agrees with $\mathcal{T}_{0}(X)$ on $\|\cdot\|_{E(X)}$-bounded sets in $E(X)$ (see $[\mathrm{W}, 2.2 .2]$ ). Since $\left(B_{E(X)}\left(2^{n}\right): n \in \mathbb{N}\right)$ is a fundamental sequence of $\gamma_{E(X)}$-bounded sets in $E(X),\left(E(X), \gamma_{E(X)}\right)$ is a generalized DF space (see [Ru, Definition 1.1]).

Recall that a locally solid topology $\tau$ on $E(X)$ is said to be uniformly Lebesgue if $f_{n} \rightarrow 0$ for $\tau$ in $E(X)$ whenever $\left\|f_{n}\right\|_{L^{0}(X)} \rightarrow 0$ with $\sup _{n}\left\|f_{n}\right\|_{E(X)}<\infty$ (see [F, Definition 2.2]).

The basic properties of $\gamma_{E(X)}$ are given in the following (see [F, Theorem 3.1]):

Proposition 2.2. We have

(i) $\gamma_{E(X)}$ is the finest uniformly Lebesgue toplogy on $E(X)$.

(ii) $f_{n} \rightarrow 0$ in $E(X)$ for $\gamma_{E(X)}$ if and only if $\left\|f_{n}\right\|_{L^{0}(X)} \rightarrow 0$ and $\sup _{n}\left\|f_{n}\right\|_{E(X)}<\infty$.

A linear operator $T: E(X) \rightarrow Y$ is said to be $\gamma$-linear if $\left\|T\left(f_{n}\right)\right\|_{Y} \rightarrow 0$ whenever $\left\|f_{n}\right\|_{L^{0}(X)} \rightarrow 0$ and $\sup _{n}\left\|f_{n}\right\|_{E(X)}<\infty$.

Proposition 2.3. For a linear operator $T: E(X) \rightarrow Y$ the following statements are equaivalent:

(i) $T$ is $\left(\gamma_{E(X)},\|\cdot\|_{Y}\right)$-continuous.

(ii) $T$ is sequentially $\left(\gamma_{E(X)},\|\cdot\|_{Y}\right)$-continuous.

(iii) $T$ is $\gamma$-linear.

(iv) $T$ is $\left(\left.\gamma_{E(X)}\right|_{B_{E(X)}(r)},\|\cdot\|_{Y}\right)$-continuous for every $r>0$.

Proof. (i) $\Rightarrow$ (ii) It is obvious.

(ii) $\Rightarrow$ (iii) It follows from Proposition 2.2(i).

(iii) $\Rightarrow$ (iv) It is obvious, because $\left.\gamma_{E(X)}\right|_{B_{E(X)}(r)}=\left.\mathcal{T}_{0}(X)\right|_{B_{E(X)}(r)}$ for $r>0$.

(iv) $\Leftrightarrow($ i) See $[\mathrm{W}, 2.2 .4]$.

Recall that a Banach space $X$ is said to be almost reflexive if every norm-bounded subset of $X$ is conditionally weakly compact (see $[\mathrm{C}],[\mathrm{H}]$ ). The fundamental $l^{1}$-Rosenthal theorem $[\mathrm{R}]$ says that $X$ is almost reflexive if and only if it contains no isomorphic copy of $l^{1}$.

From now on for a subset $H$ of $E(X)$ we will denote

$$
\widetilde{H}=\{\widetilde{f}: f \in H\} .
$$

The following result extends Proposition 1.1 to the vector-valued setting.

Proposition 2.4. Let $X$ be an almost reflexive Banach space. Then for a subset $H$ of $E(X)$ the following statements are equivalent:

(i) $\sup _{f \in H}\|f\|_{E(X)}<\infty$.

(ii) $H$ is conditionally $\sigma\left(E(X),\left(E^{\prime}\right)_{a}\left(X^{*}, X\right)\right)$-compact.

Moreover, if $X$ is a reflexive Banach space, then the statements (i)-(ii) are equivalent to the following:

(iii) $H$ is relatively $\sigma\left(E(X),\left(E^{\prime}\right)_{a}\left(X^{*}, X\right)\right)$-compact. 
Proof. (i $\Rightarrow$ (ii) Assume that $\sup _{f \in H}\|f\|_{E(X)}<\infty$, i.e., $\widetilde{H}$ is a $\|\cdot\| E^{\text {-bounded subset of }}$ $E$. Then by Proposition $1.1 \widetilde{H}$ is conditionally $\sigma\left(E,\left(E^{\prime}\right)_{a}\right)$-compact. Making use of $\left[\mathrm{N}_{4}\right.$, Corollary 2.3] we obtain that $H$ is conditionally $\sigma\left(E(X),\left(E^{\prime}\right)_{a}\left(X^{*}, X\right)\right)$-compact.

(ii) $\Rightarrow(\mathrm{i})$ Assume that $H$ is conditionally $\sigma\left(E(X),\left(E^{\prime}\right)_{a}\left(X^{*}, X\right)\right)$-compact. Then $\widetilde{H}$ is conditionally $\sigma\left(E,\left(E^{\prime}\right)_{a}\right)$-compact in $E$ (see $\left[\mathrm{N}_{4}\right.$, Theorem 2.2]), so by Proposition 1.1 $\sup _{f \in H}\|f\|_{E(X)}<\infty$.

(i) $\Leftrightarrow\left(\right.$ iii) See $\left[\mathrm{N}_{3}\right.$, Corollary 2.4$]$.

3. Weakly compact operators on Köthe-Bochner spaces. In this section we examine linear operators $T: E(X) \rightarrow Y$ whenever $E(X)$ is provided with the mixed topology $\gamma_{E(X)}$. Recall that a linear operator $T: E(X) \rightarrow Y$ is said to be $\left(\gamma_{E(X)},\|\cdot\|_{Y}\right)$-weakly compact if there exists a neighbourhood $V$ of 0 for $\gamma_{E(X)}$ such that $T(V)$ is a relatively $\sigma\left(Y, Y^{*}\right)$-compact subset of $Y$.

THEOREM 3.1. For a linear operator $T: E(X) \rightarrow Y$ the following statements are equivalent:

(i) $T$ is $\left(\gamma_{E(X)},\|\cdot\|_{Y}\right)$-weakly compact.

(ii) $T$ is $\left(\gamma_{E(X)},\|\cdot\|_{Y}\right)$-continuous and $\left(\|\cdot\|_{E(X)},\|\cdot\|_{Y}\right)$-weakly compact.

Proof. (i) $\Rightarrow$ (ii) It is obvious.

(ii) $\Rightarrow$ (i) Assume that $T$ is $\left(\gamma_{E(X)},\|\cdot\|_{Y}\right)$-continuous and $\left(\|\cdot\|_{E(X)},\|\cdot\|_{Y}\right)$-weakly compact. Hence for every $r>0, T\left(B_{E(X)}(r)\right)$ is a relatively $\sigma\left(Y, Y^{*}\right)$-compact subset of $Y$. Since $B d\left(E(X), \gamma_{E(X)}\right)=B d\left(E(X),\|\cdot\|_{E(X)}\right)$ (see Proposition 2.1), in view of [Ru, Theorem 3.1] $T$ is $\left(\gamma_{E(X)},\|\cdot\|_{E(X)}\right)$-weakly compact, as desired.

REMARK. For the proof of the implication (ii) $\Rightarrow(\mathrm{i})$ one can also use the earlier result of Grothendieck. Indeed, since $\left(E(X), \gamma_{E(X)}\right)$ is a generalized DF-space, it is as well a quasinormable Hausdorff locally convex space. Moreover, $T$ is $\left(\gamma_{E(X)},\|\cdot\|_{Y}\right)$-continuous and $\left(\|\cdot\|_{E(X)},\|\cdot\|\right)$-weakly compact, so $T$ transforms $\|\cdot\|_{E(X)}$-bounded sets into relatively $\sigma\left(Y, Y^{*}\right)$-compact sets in $Y$. Thus by [G, III. 3, Corollary 1 of Theorem 11] $T$ is $\left(\gamma_{E(X)},\|\cdot\|_{Y}\right)$-weakly compact, because $B d\left(E(X), \gamma_{E(X)}\right)=B d\left(E(X),\|\cdot\|_{E(X)}\right)$.

Corollary 3.2. Assume that a linear operator $T: E(X) \rightarrow Y$ is $\left(\|\cdot\|_{E(X)},\|\cdot\|_{Y}\right)$-weakly compact. Then the following statements are equivalent:

(i) $T$ is $\left(\gamma_{E(X)},\|\cdot\|_{Y}\right)$-weakly compact.

(ii) $T$ is $\left(\gamma_{E(X)},\|\cdot\|_{Y}\right)$-continuous.

Corollary 3.3. Assume that a linear operator $T: E(X) \rightarrow Y$ is $\left(\gamma_{E(X)},\|\cdot\|_{Y}\right)$ continuous. Then the following statements are equivalent:

(i) $T$ is $\left(\gamma_{E(X)},\|\cdot\|_{Y}\right)$-weakly compact.

(ii) $T$ is $\left(\|\cdot\|_{E(X)},\|\cdot\|_{Y}\right)$-weakly compact.

Now we are ready to present our main results.

THEOREM 3.4. Let $X$ be an almost reflexive Banach space and let $Y$ be a weakly sequentially complete Banach space. Then for a linear operator $T: E(X) \rightarrow Y$ the following 
statements are equivalent:

(i) $T$ is $\left(\gamma_{E(X)},\|\cdot\|_{Y}\right)$-continuous.

(ii) $T$ is $\left(\gamma_{E(X)},\|\cdot\|_{Y}\right)$-weakly compact.

Proof. (i) $\Rightarrow$ (ii) Assume that $T$ is $\left(\gamma_{E(X)},\|\cdot\|_{Y}\right)$-continuous. In view of Corollary 3.3 it is enough to show that $T$ transforms $\|\cdot\|_{E(X)}$-bounded sets in $E(X)$ into relatively $\sigma\left(Y, Y^{*}\right)$-compact sets in $Y$. Indeed, let $H$ be a $\|\cdot\|_{E(X)}$-bounded set in $E(X)$. Then by Proposition $2.4 H$ is conditionally $\sigma\left(E(X),\left(E^{\prime}\right)_{a}\left(X^{*}, X\right)\right)$-compact. In view of [F, Theorem 3.2] it is seen that the topology $\gamma_{E(X)}$ is coarser than the Mackey topology $\tau\left(E(X),\left(E^{\prime}\right)_{a}\left(X^{*}, X\right)\right)$. Hence $T$ is also $\left(\tau\left(E(X),\left(E^{\prime}\right)_{a}\left(X^{*}, X\right)\right),\|\cdot\|_{Y}\right)$-continuous and it follows that $T$ is $\left(\sigma\left(E(X),\left(E^{\prime}\right)_{a}\left(X^{*}, X\right)\right), \sigma\left(Y, Y^{*}\right)\right)$-continuous. Thus $T(H)$ is a conditionally $\sigma\left(Y, Y^{*}\right)$-compact subset of $Y$, and since $Y$ is $\sigma\left(Y, Y^{*}\right)$-sequentially complete, we obtain that $T(H)$ is relatively $\sigma\left(Y, Y^{*}\right)$-sequentially compact in $Y$. It follows that $T(H)$ is relatively $\sigma\left(Y, Y^{*}\right)$-compact in $Y$, as desired.

(ii) $\Rightarrow$ (i) See Theorem 3.1.

THEOREM 3.5. Let $X$ be a reflexive Banach space. Then for a linear operator $T$ : $E(X) \rightarrow Y$ the following statements are equivalent:

(i) $T$ is $\left(\gamma_{E(X)},\|\cdot\|_{Y}\right)$-continuous.

(ii) $T$ is $\left(\gamma_{E(X)},\|\cdot\|_{Y}\right)$-weakly compact.

Proof. (i) $\Rightarrow($ ii $)$ Assume that $T$ is $\left(\gamma_{E(X)},\|\cdot\|_{Y}\right)$-continuous. Since $\gamma_{E(X)} \subset \tau(E(X)$, $\left.\left(E^{\prime}\right)_{a}\left(X^{*}, X\right)\right), T$ is also $\left(\tau\left(E(X),\left(E^{\prime}\right)_{a}\left(X^{*}, X\right)\right),\|\cdot\|_{Y}\right)$-continuous. It follows that $T$ is also $\left(\sigma\left(E(X),\left(E^{\prime}\right)_{a}\left(X^{*}, X\right)\right), \sigma\left(Y, Y^{*}\right)\right)$-continuous. Note that $T$ transforms $\|\cdot\|_{E(X)^{-}}$ bounded sets in $E(X)$ into relatively $\sigma\left(Y, Y^{*}\right)$-compact sets in $Y$. Indeed, let $H$ be a $\|\cdot\|_{E(X)}$-bounded set in $E(X)$. Then by Proposition $2.4, H$ is relatively $(\sigma(E(X)$, $\left.\left(E^{\prime}\right)_{a}\left(X^{*}, X\right)\right)$-compact, and consequently $T(H)$ is relatively $\sigma\left(Y, Y^{*}\right)$-compact in $Y$. By Corollary 3.3, $T$ is $\left(\gamma_{E(X)},\|\cdot\|_{Y}\right)$-weakly compact.

(ii) $\Rightarrow$ (i) It is obvious.

\section{References}

[AB] C. D. Aliprantis and O. Burkinshaw, Locally Solid Riesz Spaces with Applications to Economics, Mathematical Surveys and Monographs 105, 2003.

[BD] O. Burkinshaw and P. Dodds, Weak sequential compactness and completeness in Riesz spaces, Canad. J. Math. 28 (1976), 1332-1339.

$\left[\mathrm{Bu}_{1}\right] \mathrm{A} . \mathrm{V}$. Bukhvalov, On an analytic representation of operators with abstract norm, Izv. Vyssh. Ucheb. Zaved. 11 (1975), 21-32.

$\left[\mathrm{Bu}_{2}\right] \quad \mathrm{A} . \mathrm{V}$. Bukhvalov, On an analytic representation of linear operators by means of measurable vector-valued functions, Izv. Vyssh. Ucheb. Zaved. 7 (1977), 21-32.

[C] R. Cross, A characterization of almost reflexivity of normed function spaces, Proc. Royal Irish Acad. Sect. A 92 (1992), 225-228.

[Co] J. B. Cooper, Saks Spaces and Applications to Functional Analysis, North-Holland, Amsterdam, 1978. 
[D] D. van Dulst, (Weakly) compact mappings into (F) spaces, Math. Ann. 224 (1976), 111-115.

[F] K. Feledziak, Uniformly Lebesgue topologies on Köthe-Bochner spaces, Comment. Math. 37 (1997), 487-511.

[G] A. Grothendieck, Sur les espaces (F) et (DF), Summa Brasil. Math. 3 (1954), 57-122.

[Ga] V. Gantmacher, Über schwache totalstetige Operatoren, Mat. Sb. (N.S.) 7 (49) (1940), 301-308.

[H] J. Howard, A generalization of reflexive Banach spaces and weakly compact operators, Comment. Math. Univ. Carolin. 13 (1972), 673-684.

[KA] L. V. Kantorovich and A. V. Akilov, Functional Analysis, 3rd ed., Nauka, Moscow, 1984 (in Russian).

[L] P.-K. Lin, Köthe-Bochner Function Spaces, Birkhäuser, Boston, 2004.

$\left[\mathrm{N}_{1}\right] \quad$ M. Nowak, Mixed topology on normed function spaces I, Bull. Acad. Polon. Sci. Math. 36 (1988), 251-262.

$\left[\mathrm{N}_{2}\right] \quad-$, Mixed topology on normed function spaces II, Bull. Acad. Polon. Sci. Math. 36 (1988), 263-267.

$\left[\mathrm{N}_{3}\right]$ M. Nowak, Weak compactness in Köthe-Bochner spaces and Orlicz-Bochner spaces, Indag. Math., N.S. 10 (1999), 73-86.

$\left[\mathrm{N}_{4}\right]$ M. Nowak, Conditional and relative weak compactness in vector-valued function spaces, J. Convex Analysis 12 (2005), 447-463.

[No 1 K. Noureddine, Espaces du type $D_{b}$, C.R. Acad. Sc. Paris 276 (1973), A1301-A1303.

$\left[\mathrm{No}_{2}\right] \mathrm{K}$. Noureddine, Nouvelles classes d'espaces localement convexes, Publ. Dept. Math. Lyon, 10 (1973), 105-123.

[R] H. Rosenthal, A characterization of Banach spaces containing $l_{1}$, Proc. Nat. Acad. Sci. USA 71 (1974), 2411-2413.

[Ru] W. Ruess, [Weakly] compact operators and DF spaces, Pacific J. Math. 98 (1982), 419-441.

[W] A. Wiweger, Linear spaces with mixed topology, Studia Math. 20 (1961), 47-68. 
